\title{
DISCRIMINANTAL DIVISORS AND BINARY QUADRATIC FORMS
}

\author{
by EZRA BROWN
}

(Received 20 April, 1970)

1. Introduction. An ancipital form is a form $[a, b, c]$ in which $b=0$ or $b=a$; these fall into pairs of associates: $[a, 0, c]$ and $[c, 0, a]$ (type 1 ), and $[a, a, c]$ and $[4 c-a, 4 c-a, c]$ (type 2). The set of discriminantal divisors of discriminant $d$ is formed by choosing, from each pair of primitive associate ancipital forms of discriminant $d$, exactly one of the two leading coefficients. In this article we study representations of discriminantal divisors of a given discriminant by binary quadratic forms of that discriminant, previously studied by the author and by G. Pall. We are concerned here with discriminants $d=4^{k} p q$, where $k \geqq 1$, $p \equiv 1, q \equiv 3(\bmod 4)$ are primes, and $d=4^{k} p$, where $k \geqq 1$ and $p$ is an odd prime. This investigation arose in connection with the search for integral solutions of $x^{2}-D y^{2}=-1$.

2. Preliminary results for the case $d=4 p q$. Suppose that $p \equiv 1, q \equiv 3(\bmod 4)$. Since $d \equiv-4(\bmod 16)$, there are the generic characters $(f \mid p),(f \mid q)$, and $(-1 \mid f)$; hence there are four genera and eight pairs of primitive associate ancipital forms. The eight discriminantal divisors ( $D D$ 's) associated with these forms turn out to be $\pm 1, \pm 2, \pm q$, and $\pm 2 q$. Now a necessary condition that $f_{1}=[1,0,-p q]$ represent $k$, a given $D D$, is that $f_{1}$ be in the genus of the ancipital form whose leading coefficient is $k$. If we construct a table of generic characters for the eight appropriate ancipital forms, we may deduce the following theorem:

THEOREM 1. Suppose that $f_{1}=[1,0,-p q]$, where $p \equiv 1, q \equiv 3(\bmod 4)$ are primes.

(a) Suppose that $(p \mid q)=-1$. Then $f_{1}$ represents 2 if $(2 \mid p)=(2 \mid q)=1,-2$ if $(2 \mid p)=-(2 \mid q)=1,2 q$ if $-(2 \mid p)=(2 \mid q)=1$, and $-2 q$ if $(2 \mid p)=(2 \mid q)=-1$.

(b) Suppose that $(p \mid q)=1$. If $(2 \mid p)=-1$, then $f_{1}$ represents $-q$; if $(2 \mid p)=1$, then $f_{1}$ represents $\{-q, 2,-2 q\}$ or $\{-q,-2,2 q\}$, according as $(2 \mid q)=1$ or -1 .

The undecided cases are $(p \mid q)=(2 \mid p)=1$; so we consider these now. In particular, we take the case $(2 \mid q)=-1$, and determine necessary conditions that $f_{1}$ represent -2 , $-q$, or $2 q$. The case $(2 \mid q)=1$ will be studied later.

THEOREM 2. Let $(p \mid q)=1$, where $p \equiv 1, q \equiv 3(\bmod 8)$ are primes; we may then write $q=A^{2}+2 B^{2}$. If $f_{1}=[1,0,-p q]$ represents -2 , then there exist integers $x_{1}$ odd, $x_{2}$ even such that $p=x_{1}^{2}+2 x_{2}^{2}$, and either

(a) $\left(A x_{2}+B x_{1} \mid q\right)=\left(-1 \mid A x_{2}+B x_{1}\right)=1$, or

(b) $\left(A x_{2}-B x_{1} \mid q\right)=\left(-1 \mid A x_{2}-B x_{1}\right)=1$.

Proof. Suppose that there exist $u, v(v>0)$ such that $u^{2}-p q v^{2}=-2$; then $g=[p q v, 2 u, v]$ has determinant 2 , and so $g \sim[1,0,2]$. Consider the following Cantor diagram (see [1]), with $\operatorname{det} T=1$ :

$$
\begin{array}{r}
{[1,0,2] \stackrel{T}{\rightarrow}[p q v, 2 u, v],} \\
h=[a, 2 b, c] \stackrel{T^{\prime}}{\leftarrow}[1,0,-p q] .
\end{array}
$$


By Proposition 3.3 of [1], $a+2 c=0$; so there is a form $h=[-2 c, 2 b, c] \sim f_{1}$; comparing determinants, we have $p q=b^{2}+2 c^{2}$. Since $(-2 \mid p)=(-2 \mid q)=1$, and $p, q$ are primes, there exist $x_{1}$ odd, $x_{2}$ even, $A$ odd, $B$ odd (unique up to choice of sign) such that $p=x_{1}^{2}+2 x_{2}^{2}$ and $q=A^{2}+2 B^{2}$. Hence $p q=\left(A x_{1} \pm 2 B x_{2}\right)^{2}+2\left(A x_{2} \mp B x_{1}\right)^{2}=b^{2}+2 c^{2}$. Since $h$ is in the genus of $f_{1},(-1 \mid c)=(c \mid q)=1$ ( $c$ is odd, since $h$ is primitive). From this the conclusion follows.

THEOREM 3. Let $p \equiv 1, q \equiv 3(\bmod 8)$ be primes. Suppose that the only classes of determinant $-2 q$ are represented by $\pm[1,0,-2 q]$. If $f_{1}$ represents $2 q$, then there exist $x_{3}, x_{4}$ both odd such that $p=q x_{4}^{2}-2 x_{3}^{2}$ and $\left(x_{3} \mid q\right)=\left(-1 \mid x_{3}\right)=1$.

Proof. If there exist $u, v$ such that $u^{2}-p q v^{2}=2 q$, then $g=[p q v, 2 u, v]$ has determinant $-2 q$; by hypothesis, $g \sim[1,0,-2 q]$ or $g \sim[-1,0,2 q]$. In either case, we have the following Cantor diagram $(\operatorname{det} T=1)$ :

$$
\begin{array}{r}
{[ \pm 1,0, \mp 2 q] \stackrel{T}{\rightarrow}[p q v, 2 u, v],} \\
{\left[a, 2 b, x_{3}\right] \stackrel{T^{\prime}}{\leftarrow}[1,0,-p q] .}
\end{array}
$$

By Proposition 3.3 of [1], $a=2 q x_{3}$; so there is a form $h=\left[2 q x_{3}, 2 b, x_{3}\right] \sim f_{1}$; comparing determinants, we have $p q=b^{2}-2 q x_{3}^{2}$. Hence $b=q x_{4}, p=q x_{4}^{2}-2 x_{3}^{2}$; since $h$ is primitive and $p q$ is odd, $x_{3}$ and $x_{4}$ are both odd, and since $h$ is in the genus of $f_{1},\left(-1 \mid x_{3}\right)=\left(x_{3} \mid q\right)=1$.

REMARK. The hypothesis that there be only two classes of determinant $-2 q$ is not strong; the smallest prime $q \equiv 3(\bmod 8)$ not having this property is 163 .

As in the case $p \equiv q(\bmod 4)$, the necessary conditions that $f_{1}$ represent $-q$ depend upon the class number $h(q)$ of determinant $q$; if $h(q)$ is large, these necessary conditions may be complicated. However, we may prove the following general theorem.

THEOREM 4. Let $p \equiv 1, q \equiv 3(\bmod 8)$ be primes, and suppose that $u^{2}-p q v^{2}=-q . \quad$ Let $g=[p q v, 2 u, v]$ and $g_{1}=[1,0, q]$. If $g \sim g_{1}$, then there exist $x_{5}$ odd, $x_{6}$ even such that $p=x_{5}^{2}+q x_{6}^{2}$, and $\left(x_{5} \mid q\right)=\left(-1 \mid x_{5}\right)=1$.

Proof. The result follows from the Cantor diagram $(\operatorname{det} T=1)$ :

$$
\begin{aligned}
& {[1,0, q] } \stackrel{T}{\rightarrow}[p q v, 2 u, v], \\
& h=\left[a, 2 b, x_{5}\right] \stackrel{T^{\prime}}{\leftarrow}[1,0,-p q] .
\end{aligned}
$$

As in [1], we find it useful to study a system of diophantine equations in order to discern any relationships among the forms discussed in Theorems 2,3 , and 4 . We study the system

$$
p=x_{1}^{2}+2 x_{2}^{2}=q x_{4}^{2}-2 x_{3}^{2}=x_{5}^{2}+q x_{6}^{2}
$$

in the case $x_{1}, x_{3}, x_{4}, x_{5}$ odd, $x_{2}, x_{6}$ even, $p \equiv 1(\bmod 8)$ and $q \equiv 3(\bmod 8)$ primes, and $p$ representable by $x_{1}^{2}+2 x_{2}^{2}$ and $x_{5}^{2}+q x_{6}^{2}$.

First, we study the solutions of

$$
x_{1}^{2}+2 x_{2}^{2}=q x_{4}^{2}-2 x_{3}^{2}
$$

in the above case. We see that $Q=x_{1} i_{1}+x_{2} i_{2}+x_{3} i_{3}$ is an element of norm $q x_{4}^{2}$ in the ring 
of generalized quaternions with multiplication $i_{1}^{2}=-1, i_{2}^{2}=i_{3}^{2}=i_{1} i_{2} i_{3}=-2$. The norm form of this ring, $x^{2}+y^{2}+2 z^{2}+2 w^{2}$, is in a genus of one class (see [3]); as a consequence of this and Theorem 3 of [3] we may write $Q=\bar{\sigma} \tau \sigma$, where $N(\tau)=q, N(\sigma)=x_{4}$, and $\sigma$ and $\tau$ are unique up to multiplication by unit factors (see [1] for elaboration). Since $q$ is a prime $\equiv 3$ $(\bmod 8)$, there exist $A, B$, both odd, such that $q=A^{2}+2 B^{2}$, where $A$ and $B$ are unique up to choice of sign. It is not hard to show that, if $\tau=a i_{1}+b i_{2}+c i_{3}$, then $a \equiv x_{1}, b \equiv x_{2}$ and $c \equiv x_{3}(\bmod 2)$; hence the only possibilities for $\tau$ are $\pm\left(A i_{1} \pm B i_{3}\right)$. If we use $\tau_{1}=A i_{1}+B i_{3}$, write $\sigma=s_{0}+s_{1} i_{1}+s_{2} i_{2}+s_{3} i_{3}$, and expand $\bar{\sigma} \tau_{1} \sigma$, we obtain the following expressions:

$$
\left.\begin{array}{l}
x_{1}=A\left(s_{0}^{2}+s_{1}^{2}-2 s_{2}^{2}-2 s_{3}^{2}\right)+4 B\left(-s_{0} s_{2}+s_{1} s_{3}\right), \\
x_{2}=2 A\left(-s_{0} s_{3}+s_{1} s_{2}\right)+2 B\left(s_{0} s_{1}+2 s_{2} s_{3}\right), \\
x_{3}=2 A\left(s_{0} s_{2}+s_{1} s_{3}\right)+B\left(s_{0}^{2}+2 s_{3}^{2}-s_{1}^{2}-2 s_{2}^{2}\right), \\
x_{4}=s_{0}^{2}+s_{1}^{2}+2 s_{2}^{2}+2 s_{3}^{2} \quad\left(\text { where } s_{0} \neq s_{1}(\bmod 2)\right) .
\end{array}\right\}
$$

It is straightforward to show that, if we replace $\tau_{1}$ by one of the other three eligible $\tau$ 's, we gain no new solutions; hence all parametric solutions of (2) are given by the expressions (3).

Consider the following expressions for $x_{5}$ and $x_{6}$, obtained by considering special cases:

$$
\left.\begin{array}{l}
x_{5}=A\left(s_{0}^{2}+2 s_{3}^{2}-s_{1}^{2}-2 s_{2}^{2}\right)+4 B\left(-s_{0} s_{2}-s_{1} s_{3}\right), \\
x_{6}=2\left(s_{0} s_{1}-2 s_{2} s_{3}\right) .
\end{array}\right\}
$$

The expressions in (3) and (4), when substituted into the following equations, yield an identity:

$$
x_{1}^{2}+2 x_{2}^{2}=q x_{4}^{2}-2 x_{3}^{2}=x_{5}^{2}+q x_{6}^{2} .
$$

Since the expressions in (3) yield all solutions of (2), and since the representations of a prime by the forms $x_{1}^{2}+2 x_{2}^{2}$ and $x_{5}^{2}+q x_{6}^{2}$ ( $q$ a prime) are essentially unique, it follows that all solutions of (1) in the stated case are given by the parametric expressions for $x_{1}, \ldots, x_{6}$ in (3) and (4). The key to the solution of this system is that the norm-form $x^{2}+y^{2}+2 z^{2}+2 w^{2}$ is in a genus of one class; hence the factorization of $Q$ as $\bar{\sigma} \tau \sigma$ given above is essentially unique.

3. The main theorem, for $q=3$. First, we prove

THEOREM 5. Suppose that $p \equiv 1(\bmod 8),(p \mid 3)=1$, and $f_{1}=[1,0,-3 p]$. If $f_{1}$ represents -3 , then (a) there exist $x_{5}$ odd, $x_{6}$ even such that $p=x_{5}^{2}+3 x_{6}^{2}$. Furthermore, (b) $x_{5} \equiv \pm 1$ $(\bmod 6)$ and $x_{6} \equiv 0(\bmod 4)$.

Proof. Let $u^{2}-3 p v^{2}=-3$; then $g=[3 p v, 2 u, v]$ has determinant 3. If $g \sim[1,0,3]$, then (a) is true by Theorem 4 . If $g \sim[2,2,2]$ (the only other possibility), we deduce that there is a form $h=[-b-c, 2 b, c] \sim f_{1}$, by examining the following Cantor diagram, in which $\operatorname{det} T=1$ :

$$
\begin{array}{r}
{[2,2,2] \stackrel{T}{\rightarrow}[3 p v, 2 u, v],} \\
h=[a, 2 b, c] \stackrel{T^{\prime}}{\leftarrow}[1,0,-3 p] .
\end{array}
$$


Hence $3 p=b^{2}+b c+c^{2}$; one of $b, c$ is odd; so we suppose in view of the symmetry that $b$ is odd. We may assume that $c$ is even, for if $c$ is also odd, we can replace $c$ by $b+c$ and $b$ by $-b$. Writing $c=2 x_{5}$, we obtain $3 p=\left(b+x_{5}\right)^{2}+3 x_{5}^{2}$; writing $b+x_{5}=3 x_{6}$, we obtain $p=x_{5}^{2}+3 x_{6}^{2}$. By hypothesis, $p \equiv 1(\bmod 24)$, so we must have $x_{5}$ odd and $x_{6}$ even. To prove (b) in either case, we observe that $(p, 3)=1$ and so $x_{5} \equiv \pm 1(\bmod 6)$; hence $x_{5}^{2} \equiv 1(\bmod 24)$ and so $x_{6} \equiv 0(\bmod 4)$.

Now we may prove

THEOREM 6. Let $p \equiv 1(\bmod 8),(p \mid 3)=1, f_{1}=[1,0,-3 p]$, and let $x_{1}, \ldots, x_{6}$ be as in equation (1).

(a) If $x_{5} \equiv \pm 5(\bmod 12)$, then $f_{1}$ never represents -3 ; it represents 6 or -2 , according as $\pm x_{3} \equiv 1$ or $5(\bmod 12)$, or equivalently, according as $\pm\left(x_{1}+x_{2}\right) \equiv 5$ or $1(\bmod 12)$.

(b) If $x_{5} \equiv \pm 1(\bmod 12)$, then $f_{1}$ represents -3 if $\pm x_{3} \equiv 5(\bmod 12)$; otherwise, any of $-2,-3$, or 6 may be represented.

The proof is based on the following lemma. Here, $x_{1}, \ldots, x_{6}, s_{0}, \ldots, s_{3}$ are as in (3) and (4).

Lemma 6.1. (a) If $x_{5} \equiv \pm 5(\bmod 12)$, then $x_{3} \equiv \pm 1(\bmod 12)$ if and only if $x_{1}+x_{2} \equiv \pm 5$ $(\bmod 12)$.

(b) If $x_{5} \equiv \pm 1(\bmod 12)$, then $\pm x_{3} \equiv x_{1}+x_{2}(\bmod 12)$.

Proof. We shall prove (a) in the case $s_{2} \equiv s_{3}(\bmod 2)$. The proofs for the case $s_{2} \not \equiv s_{3}$ $(\bmod 2)$ and for $(b)$ are similar.

Assume that $s_{2} \equiv s_{3}(\bmod 2)$. We observe that $x_{5} \equiv\left(s_{0}-2 s_{2}\right)^{2}-\left(s_{1}+2 s_{3}\right)^{2}(\bmod 12)$. If $x_{5} \equiv \pm 5(\bmod 12)$, then either (i) $s_{0}-2 s_{2} \equiv \pm 2, s_{1}+2 s_{3} \equiv 3(\bmod 6)$, or (ii) $s_{0}-2 s_{2} \equiv 3$, $s_{1}+2 s_{3} \equiv \pm 2(\bmod 6)$. Similarly, we observe that, if $x_{3} \equiv \pm 1(\bmod 12)$, then either (i) $s_{0}+s_{2} \equiv \pm 1, s_{1}-s_{3} \equiv 0(\bmod 6)$, or (ii) $s_{0}+s_{2} \equiv 0, s_{1}-s_{3} \equiv \pm 1(\bmod 6)$. Then we observe that $\pm\left(x_{1}+x_{2}\right) \equiv\left(\left(s_{0}-2 s_{2}\right)+\left(s_{1}+2 s_{3}\right)\right)^{2}+6\left(s_{0} s_{3}+s_{1} s_{2}\right)(\bmod 12)$, so that $x_{1}+x_{2} \equiv \pm 5$ $(\bmod 12)$ implies that $s_{0} s_{3}+s_{1} s_{2}$ is odd and $\left(s_{0}-2 s_{2}\right)+\left(s_{1}+2 s_{3}\right) \equiv \pm 1(\bmod 6)$. Finally, if $x_{5} \equiv \pm 5(\bmod 12)$, we observe that (i) $s_{0} \not s_{1}, s_{2} \equiv s_{3} \equiv 1(\bmod 2)$, (ii) $x_{3} \equiv \pm 1(\bmod 12)$, and (iii) $x_{1}+x_{2} \equiv \pm 5(\bmod 12)$ are equivalent statements. This proves (a) in the case $s_{2} \equiv s_{3}$ $(\bmod 2)$.

Proof of the theorem. (a) If $x_{5} \equiv \pm 5(\bmod 12)$, the necessary conditions of Theorem 5 for $f_{1}$ to represent -3 are violated; hence $f_{1}$ does not represent -3 . By the lemma, the conditions of Theorem 2 for $f_{1}$ to represent -2 are violated if $x_{3} \equiv \pm 1(\bmod 12)$, and those of Theorem 3 for $f_{1}$ to represent 6 are violated if $x_{3} \equiv \pm 5(\bmod 12)$. This proves (a).

(b) If $x_{5} \equiv \pm 1(\bmod 12)$, and $x_{3} \equiv \pm 5(\bmod 12)$, then, by the lemma and Theorems 2 and $3, f_{1}$ represents neither -2 nor 6 ; hence $f_{1}$ represents -3 . If $x_{3} \equiv \pm 1(\bmod 12)$, the following are examples demonstrating the latter statement of (b): $[1,0,-3 p]$ represents $-2,-3$, and 6 , respectively, when $p=937,433$, and 673 , respectively.

4. The cases $d=4^{k} p q$ and $d=4^{k} p(k \geqq 1)$.

THEOREM 7. Let $p \equiv 1, q \equiv 3(\bmod 8)$ be primes; let $f_{k}=\left[1,0,-4^{k-1} p q\right]$ be the principal form of discriminant $4^{k} p q$. 
(a) If $f_{1}$ represents any of $2,-2,2 q$, or $-2 q$, or if $(p \mid q)=-1$, then $f_{k}$ represents $4^{k-1}$, where $k \geqq 2$.

(b) Let $u^{2}-p q v^{2}$ be a primitive representation of $-q$, and write $v=2^{m} v_{0}$, where $v_{0}$ is odd. Let $k \geqq 2$. Then $f_{k}$ represents $-4^{k-1} q$ if $m=0,4$ if $0<m<k-3,-4 q$ if $m=k-2$, and $-q$ if $m \geqq k-1$.

Proof. By examining tables of generic characters, we find that, for $d=16 p q$, the $D D$ 's that $f_{2}$ may represent are $-q, 4$, and $-4 q$, and for $d=4^{k} p q(k \geqq 3)$ those that $f_{k}$ may represent are $-q, 4,-4 q, 4^{k-1}$, and $-4^{k-1} q$.

Suppose that $f_{2}$ represents $-q$; for some $u, v$ with $(u, v)=1$, we have $u^{2}-4 p q v^{2}=-q$. Hence $u$ is odd, and $u^{2}-p q(2 v)^{2}=-q$ is a primitive representation of $-q$ by $f_{1}$. Similarly, if $f_{2}$ represents $-4 q$, then $f_{1}$ represents $-q$ with $u$ even. Hence, if $f_{1}$ represents any of \pm 2 , $\pm 2 q$ (which happens if $(p \mid q)=1$, by Theorem 1), then $f_{2}$ represents neither $-q$ nor $-4 q$, and hence represents 4 . If there exist $u, v$ with $(u, v)=1$, such that $u^{2}-4 p q v^{2}=4$, then $u$ is even; so $\left(2^{k-2} u\right)^{2}-4^{k-1} p q v^{2}=4^{k-1}$ is a primitive representation of $4^{k-1}$ by $f_{k}(k \geqq 3)$, which proves (a).

Suppose that $u^{2}-p q v^{2}=-q$, with $(u, v)=1$. Write $v=2^{m} v_{0}$, where $v_{0}$ is odd. If $m \geqq k-1$, then $u^{2}-4^{k-1} p q\left(2^{m-k+1} v_{0}\right)^{2}=-q$, with $\left(u, 2^{m-k+1} v_{0}\right)=1$. If $m=k-2$, then $u^{2}-4^{k-2} p q v_{0}^{2}=-q$, with $u$ odd, and $\left(u, v_{0}\right)=1$; so $(2 u)^{2}-4^{k-i} p q v_{0}^{2}=-4 q$, with $\left(2 u, v_{0}\right)=1$. If $m=0$, then $\left(2^{k-1} u\right)^{2}-4^{k-1} p q v_{0}^{2}=-4^{k-1} q$, with $\left(2^{k-1} u, v_{0}\right)=1$. Conversely, if $f_{k}$ represents $-q,-4 q$, or $-4^{k-1} q$, then $u^{2}-p q\left(2^{m} v_{0}\right)^{2}=-q$, with $m \geqq k-1, m=k-2$, or $m=0$, respectively. Hence $0<m<k-3$ implies that $f_{k}$ represents 4 , which proves (b).

Using the same techniques, we prove

THEOREM 8. Let $p$ be an odd prime. Let $g_{k}=\left[1,0,-4^{k-1} p\right]$, where $k \geqq 2$. Then $g_{k}$ represents $-4^{k-1}$ or $4^{k-1}$, according as $p \equiv 1$ or $3(\bmod 4)$. Also, $[1,0,-p]$ represents -1 if $p \equiv 1(\bmod 4),-2$ if $p \equiv 3(\bmod 8)$, and 2 if $p \equiv 7(\bmod 8)$.

The proof is immediate if one realizes that the discriminant $4 p$ has one or two primitive genera, according as $p \equiv 1$ or $3(\bmod 4)$, and that, in any case, $[1,0,-4 p]$ must represent 4 .

\section{REFERENCES}

1. Ezra Brown, Representations of discriminantal divisors by binary quadratic forms, J. Number Theory 3 (1971), 213-225.

2. Gordon Pall, On generalized quaternions, Trans. Amer. Math. Soc. 59 (1946), 280-332.

3. Gordon Pall, Discriminantal divisors of binary quadratic forms, J. Number Theory 1 (1969), 525-532.

Virginia Polytechnic Institute and State University BLACKSBURG, VIRGINIA 24061 